\title{
Treatment of small cell lung cancer by pneumonectomy and single course high dose chemotherapy
}

\author{
T J LITTLEWOOD, A P SMITH, D P BENTLEY \\ From the Departments of Haematology and Thoracic Medicine, Llandough Hospital, Penarth, South Glamorgan
}

It is generally accepted that the treatment of choice for patients with small cell lung cancer is combination chemotherapy, after which up to $80 \%$ of patients will achieve a complete or partial response. ${ }^{1}$ Most of these patients will, however, eventually relapse and die from the effects of disseminated tumour. Because of the low cure rate in patients with this disease, new approaches to treatment are important. In this paper we describe two patients with small cell lung cancer who were treated with a total pneumonectomy followed by high dose chemotherapy and autologous bone marrow transplantation.

\section{PATIENT 1}

A 44 year old man presented with a two month history of malaise and a dry cough. A chest radiograph showed a $9 \times 7 \mathrm{~cm}$ mass in the right lower lobe and a diagnosis of small cell lung cancer was made after bronchoscopic biopsy of the tumour. Staging procedures included full blood count and biochemical profile, an ultrasound abdominal scan, isotope brain scan, bone marrow aspiration, and trephine biopsy; they yielded no evidence of disseminated disease. The tumour was considered operable and a right pneumonectomy was performed. Histological examination of the resected specimen showed tumour deposits in the hilar lymph nodes.

One month after the operation the patient was referred for chemotherapy. A repeat of the staging procedures detected no tumour. One day before he started chemotherapy, bone marrow was harvested from the patient under general anaesthesia and preserved in liquid nitrogen. A Nutricath S catheter was inserted into the superior vena cava during the anaesthetic. Treatment was with cyclophosphamide 100 $\mathrm{mg} / \mathrm{kg}$ and etoposide $1400 \mathrm{mg} / \mathrm{m}^{2}$. Cyclophosphamide was given by slow intravenous bolus injection in divided doses on each of three days accompanied by 2-mercaptoethane sulphonate sodium (Mesna). ${ }^{2}$ Etoposide was dissolved in $0.9 \%$ sodium chloride at a concentration not exceeding 0.5 $\mathrm{mg} / \mathrm{ml}$ sodium chloride and infused over eight hours on days 1-3 inclusive. Bone marrow was reinfused 96 hours after the completion of chemotherapy. A period of pancytopenia followed chemotherapy but blood counts had returned to normal by 28 days after treatment. Toxicity effects consisted of alopecia, which was total by day 14 , and mild nausea and vomiting, which was readily controlled by antiemetics. The patient was discharged home 21 days after the start of treatment and returned to a normal lifestyle with no restriction of

Address for reprint requests: Dr TJ Littlewood, Department of Haematology, Hammersmith Hospital, London W12 0HS.

Accepted II July 1986 activities. One hundred and eighteen weeks after diagnosis he was readmitted as an emergency complaining of chest pain. He died within 24 hours. At necropsy recurrent small cell lung cancer was found in the mediastinal lymph nodes with invasion of the pericardium by tumour.

\section{PATIENT 2}

A 33 year old symptomless man who worked as a miner at the coal face attended the pneumoconiosis board for a chest radiograph. This revealed a $10 \times 8 \mathrm{~cm}$ mass in the left lower lobe and a diagnosis of small cell lung cancer was made after bronchoscopic biopsy of the tumour. Staging procedures (as described for patient 1) detected no metastatic tumour and a left pneumonectomy was performed. Histological examination of the resected sample showed no tumour in the hilar lymph nodes. The patient was referred for high dose chemotherapy with autologous bone marrow transplantation. A repeat of the staging procedures detected no tumour and treatment was given as for patient 1. After an uneventful recovery from the chemotherapy the patient was entirely well for 80 weeks, at the end of which enlarged cervical lymph nodes were noted. Biopsy of one of these lymph nodes showed infiltration with small cell cancer. Treatment with combination chemotherapy that included etoposide was given and the patient has achieved a complete remission.

\section{Discussion}

The median survival of patients with untreated small cell lung cancer is about 10 weeks, ${ }^{3}$ whereas after combination chemotherapy the median survival is 14 months for patients with limited disease (tumour confined to one hemithorax and ipsilateral supraclavicular lymph nodes) and seven months for patients with extensive disease (tumour spread beyond the hemithorax and ipsilateral supraclavicular lymph nodes). ${ }^{1}$ After chemotherapy about $15-20 \%$ of patients with limited disease can expect to survive for more than three years. ${ }^{1}$ The long term survival figures for patients treated with either radiotherapy or surgery ${ }^{4}$ are significantly less good and chemotherapy is considered the treatment of choice.

In this paper we describe two patients with limited small cell lung cancer who were treated in a novel and aggressive way by total pneumonectomy followed, within one month, by a single course of very high dose chemotherapy. Total pneumonectomy removes the primary site, which is the commonest site of relapse in patients treated with chemotherapy who achieve a complete response, ${ }^{5}$ and it greatly reduces the overall tumour mass. Although patient 1 had tumour detected in the resected hilar lymph nodes, both patients would have been considered as having achieved complete 
remission on the basis of the staging procedures described. In these circumstances complete eradication of the tumour by means of intensive high dose chemotherapy with two agents known to be effective in small cell lung cancer seemed possible. This did not occur and it is uncertain whether chemotherapy prolonged life.

The reasons for this failure may be multiple but the most likely explanations are either that tumour cells were present in sites that were inaccessible to the cytotoxic agents or that there was a group of tumour cells with intrinsic resistance to both cyclophosphamide and etoposide-or both explanations might apply. High dose cyclophosphamide and etoposide have been investigated as late intensification treatment of patients previously treated with combination chemotherapy. ${ }^{6}$ In this trial etoposide and cyclophosphamide were used in the induction treatment, and it was unclear whether the poor results obtained by the late intensification were due to intrinsic or acquired drug resistance. The results from our two patients suggest that intrinsic drug resistance is the major problem and that high dose chemotherapy with the combination used here cannot overcome this problem even when there is only a small tumour cell population to be eradicated.

Although this treatment protocol had the advantage of a short treatment time, total pneumonectomy and high dose chemotherapy is an arduous treatment regimen that could not be justified in most patients with small cell lung cancer unless they could be offered a reasonable hope of cure or survival, superior to that obtained with conventional treat ment. We have been unable to demonstrate such benefit in these two patients, although in a larger study significantly better (or significantly worse) results might conceivably occur.

\section{References}

1 Aisner J, Alberto P, Bitran J, et al. Role of Chemotherapy i $\vec{P}$ small cell lung cancer: a consensus report of the Internationat Association for the Study of Lung Cancer Workshop. Canceft Treat Rep 1983;67:37-43.

2 Bryant BM, Jarman M, Ford HT, Smith IE. Prevention o isophosphamide-induced urothelial toxicity with 2-mercaptoethane sulphonate sodium (Mesnum) in patient with advanced carcinoma. Lancet 1980;ii:657-9.

3 Hyde L, Yee J, Wilson R. Cell type and the natural history op lung cancer. JAMA 1965;193:53-4.

4 Fox W, Scadding JG. Medical Research Council comparative trial of surgery and radiotherapy for primary treatment of small celled or oat celled carcinoma of the bronchus. Ten year follow up. Lancet 1973;ii:63-5.

5 Byhardt RW, Libnoch JA, Cox JD, et al. Local control of intra thoracic disease with chemotherapy and role of prophylactie cranial irradiation in small cell carcinoma of the lung. Cancees 1982;47:2239-46.

6 Cunningham D, Barnham SW, Hutcheon AH, et al. High dose cyclophosphamide and VP 16 as late dose intensification thero apy for small cell carcinoma of the lung. Cancer Chemother Pharmacol 1985;15:303-6. 